\title{
Kernos
}

Revue internationale et pluridisciplinaire de religion grecque antique

9 | 1996

Varia

\section{Politique et religion à Éphèse entre 550 et 450}

\section{Jean Frère}

URL : http://journals.openedition.org/kernos/1158

DOI : 10.4000/kernos.1158

ISSN : 2034-7871

\section{Éditeur}

Centre international d'étude de la religion grecque antique

Édition imprimée

Date de publication : 1 janvier 1996

ISSN : 0776-3824

\section{Référence électronique}

Jean Frère, «Politique et religion à Éphèse entre 550 et 450 », Kernos [En ligne], 9 | 1996, mis en ligne le 21 avril 2011, consulté le 19 avril 2019. URL : http://journals.openedition.org/kernos/1158 ; DOI :

$10.4000 /$ kernos. 1158 


\section{Politique et religion à Éphèse entre 550 et 450}

La période qui va des années 550 aux années 450 constitue pour Éphèse une période cruciale. Certes, Éphèse reste, comme dans les périodes antérieures ou postérieures, tributaire d'une double influence, celle de l'Asie, celle d'Athènes et de la Grèce. Mais, aussi bien politiquement que religieusement, Éphèse se trouve affrontée à des changements d'importance. Or cette période trouve un témoin essentiel en la personne du philosophe Héraclite (560-470 environ). Éphèse à cette époque connaît successivement plusieurs régimes politiques. Nous en retiendrons deux : l'époque des tyrans, l'époque d'un régime démocratique; s'y ajoutera un troisième moment : celui où se constitue le mouvement idéologique d'une royauté idéale telle que la proposent certains écrits d'Héraclite. À ces trois régimes politiques correspondent du point de vue religieux des conceptions ayant évolué de façon sensiblement parallèle. Représentations politiques et représentations religieuses d'inspiration tour à tour comparable vont surgir aux trois moments ici envisagés : le moment historique des tyrans, le moment historique des démocrates, le moment de la royauté idéale selon Héraclite.

Les sources ici retenues seront donc de plusieurs types: sources historiques (Hérodote, Élien, Strabon, etc), sources philosophiques : les fragments d'Héraclite.

Il y a d'abord l'époque des tyrans d'Éphèse; ce sont successivement Pindaros, Athénagoras, Comas, Aristarchos, Pythagoras, Mélancomas. C'est une période marquée par la tutelle de l'Asie : d'abord celle de la Lydie avec Crésus (561-546), puis celle de la Perse, avec Cyrus le Grand (559-529), avec Darius (522-486), avec Xerxès (486-465), bref avec les grands Achéménides. C'est aussi bien l'époque où s'impose à Éphèse la grande déesse Artémis, qu'on vénère à l'Artémision, et qui éclipse toutes les autres divinités.

Vient, après l'époque des tyrans, la mise en place d'un régime démocratique plus ou moins inspiré de l'Athènes de Clisthène. Dans cette période qui se déroule d'abord au lendemain de la révolte manquée des cités d'Ionie révolte où Éphèse n'intervient guère (494) - puis, au lendemain des grandes défaites perses de Marathon (490) et de Salamine (480), Éphèse se donne un gouvernement populaire lequel n'exclut point certaines tensions et agitations. Cette période, où subsiste l'influence perse mais où s'affirme davantage l'influence de la Grèce démocratique, voit corrélativement se développer des conceptions religieuses apportées de Grèce, conceptions selon lesquelles les dieux passionnés et hiérarchisés d'Homère sont remplacés par des dieux 
qu'égalise le souci de la justice, de la Diké, conceptions religieuses qui s'accordent bien avec l'établissement d'une constitution de type démocratique.

Face à l'époque historique des tyrans et à l'époque historique des démocrates, se présente un troisième moment : celui de la double critique héraclitéenne. Cette critique porte contre les régimes tyranniques ou contre les régimes démocratiques ainsi que contre les multiples formes du paganisme; s'y oppose le projet théorique esquissé d'une cité royale idéale.

\section{L'époque des tyrans d'Éphèse}

Le caractère essentiel des tyrans d'Tonie et des îles (Pythagoras à Éphèse, Thrasyboulos à Milet, Polycrate à Samos, Lygdamis à Naxos), c'est d'avoir été les ennemis acharnés d'une grande partie de l'aristocratie. Le tyran, dit Aristote, fait une guerre continuelle aux riches. Le tyran, commente Gustave Glotz, c'est avant tout le chef qui mène la multitude contre les riches, la roture contre la noblesse, celui que la foule met spécialement à sa tête et qu'elle travaille à rendre plus grand et plus puissant ${ }^{2}$. Il n'y eut de tyrans que dans les villes où le peuple fut assez nombreux et assez fort pour s'organiser, dans les États où le régime agricole avait fait place à l'économie industrielle et commerciale. Thucydide voit une étroite corrélation entre l'établissement de la tyrannie et l'accroissement de la richesse ${ }^{3}$. À Éphèse comme dans les autres cités ioniennes, les tyrans durent le pouvoir au peuple autant qu'à leur audace. C'étaient des démagogues ${ }^{4}$. Les tyrans n'abolissent pas les lois ordinaires. Ils interdisent le port d'armes aux simples citoyens; ils respectent en apparence les institutions traditionnelles et tout spécialement les divinités nationales. Ils développent le commerce. Ils exécutent les travaux d'utilité publique et d'embellissement; ils facilitent la vie matérielle à toute la population. Ils endorment le désir de liberté par des satisfactions de fierté civique. Ils attachent leur nom à des monuments impérissables. Tels les princes italiens de la Renaissance, ils se piquent d'émulation à la recherche des talents. Ils s'efforcent d'assurer la paix : y contribuent l'entraide entre tyrans et une politique de mariage pour ménager l'indépendance de la cité.

Pour ce qui est de la politique extérieure, considérons, à l'époque où Éphèse dépend encore de la Lydie (milieu du VIe siècle), comment va se constituer un complot au moment de l'accession au trône du fils de l'avantdernier successeur de Gygès, Alyatte (610-561). Ce fils d'Alyatte de Lydie qui allait accéder au trône, c'est Crésus. Or le tyran qui dominait alors Éphèse, Pindaros, aurait préféré voir monter sur le trône de Sardes, non pas Crésus, mais

\footnotetext{
ARISTOTE, Politique, V, 1311a 27-28; VIII, 8, 7.

PLATON, République, VIII, 568c.

3 THUCYDIDE, I, $13,1$.

4 ARISTOTE, Politique, V, $1310 \mathrm{~b} 15 ; \mathrm{VIII}, 8,2-3$.
} 
le frère de celui-ci, Pantaléon ${ }^{5}$, Crésus l'emporta. À peine Roi, Crésus se hâta de se venger de ceux qui avaient machiné sa perte. Des ambassadeurs invitèrent Pindaros à la soumission. Comme Pindaros, homme énergique, patriote intraitable et violent ${ }^{6}$ se refusait à toute déchéance, une armée conduite par Crésus parut aux bouches du Caystre. Les murailles de la ville ayant été mises à mal à la suite de la chute d'une tour, Pindaros dut négocier la capitulation.

C'est ici que se fait voir le lien profond entre considérations religieuses et considérations politiques. Pour obtenir plus aisément la clémence de Crésus le Lydien, que fit Pindaros l'Éphésien ? Son flair subtil de Grec ionien, observateur pratique, habile à tirer parti des circonstances les plus fâcheuses, lui suggéra un stratagème. Pindaros avait pénétré à fond le caractère de son oncle, sa bonbomie dévote de barbare superstitieux $x^{7}$, sa crédulité pour les oracles, son culte pour les sanctuaires en renom. En conséquence, Pindaros persuada les Éphésiens de consacrer leur ville à leur grande divinité tutélaire, Artémis, et, pour cela, de réunir au moyen d'une corde la citadelle au temple, lequel était situé beaucoup plus bas en rase campagne ${ }^{8}$. Pindaros alors envoya une ambassade à Crésus et lui demanda la paix au nom de la Déesse. Crésus, amusé par la ruse des habitants ${ }^{9}$, intimidé par la crainte de commettre un sacrilège s'il continuait à menacer une propriété divine ${ }^{10}$, se laissa fléchir. Moyennant certaines charges fiscales et militaires qui pesèrent uniformément sur toute l'Ionie, Crésus garantit par un acte solennel l'autonomie de la cité. C'est alors seulement que Pindaros fut expulsé et partit pour le Péloponnèse.

Or comment se représenter la grande déesse Artémis que vénérèrent les Éphésiens et leurs tyrans aussi bien que les Lydiens? Le nom d'Artémis est assurément grec. Mais la grande déesse d'Éphèse possède des attributs originaux et davantage perses que grecs. Ainsi que l'a montré Charles Picard, le rôle des Lydiens paraît avoir été considérable dans la période où s'installait l'Artémis d'Éphèse. Le premier culte s'était adressé à une «anassa », Opis ou Oupis, la Dame ou la Reine. Au demeurant, ce type de déesse aurait aussi remplacé, à Sardes même, la déesse Cybèle ${ }^{11}$. C'était déesse de la fécondité tout autant que déesse de la chasse. Les figurines de l'Éphesia comportent ces deux types. Il y a la "potnia therôn » représentée entre deux lions menaçants dressés face à face de chaque côté d'elle et de taille très supérieure à celle de l'idole; la déesse semblait armée d'un poignard ${ }^{12}$. Il y a la déesse aux éperviers.

5 HÉRODOTE, I, 92, 4.

6 ÉLIEN, Histoires variées, III, 26.

7 G. RADET, La Lydie et le monde grec au temps des Mermnades, Paris, 1892.

8 HÉRODOTE, I, 26, 2

9 ELIEN, Histoires variées, III, 26.

10 POLYEN, Stratagèmes, VI, 50.

11 XÉNOPHON, Anabase, I, 6, 7.

12 Ch. PICARD, Epbèse et Claros, Paris, 1922, p. 479. 
Il y a la déesse tenant une lyre en écaille de tortue ${ }^{13}$. Mais il y a aussi la figurine aux seins multiples, seins il est vrai postiches, puisque la déesse est vêtue par dessous. Ces faux seins seraient des ornements sacrés, dérivant sans doute du plastron à ornements saillants de divinités babyloniennes ${ }^{14}$. Le culte de ces divinités était culte répandant l'abondance. Culte confié au Grand prêtre eunuque à la dénomination perse : le « mégabise ».

Ainsi l'Artémis éphésienne se révèle-t-elle très proche, en son symbolisme religieux multiple, des représentations symboliques du pouvoir des tyrans: aussi bien tyrans d'Éphèse ou tyrans de Lydie, qu'un peu plus tard despotes de Perse. Par exemple en Lydie on trouve le culte de l'épervier, oiseau sacré; or en 687 , après la régression des cultes de la double hache et du lion, attributs sacrés des Héraclides, Gygès « l'orfraie » instaure la dynastie des Mermnades, les rois-éperviers ${ }^{15}$. Quant à l'aspect de la divinité comme force vitale, créatrice de la sagesse, maîtresse du destin des hommes de l'Artémis d'Éphèse, on trouvera tous ces aspects chez les Perses à la fois dans la figure des dieux du mazdéisme aussi bien que dans la figure du Grand Roi. C'est ainsi qu'il y a d'abord Ahura-Mazda, créateur de l'univers, de la sagesse, et force vitale de la nature. Il y a aussi Mithra, l'alter ego de l'Ahura-Mazda, commandant à la vie des hommes et à celle des dieux.

Par conséquent, entre la toute puissance protectrice qui préside aux desseins d'Ahura-Mazda ou à ceux de Cyrus, de Darius, de Xerxès - et la toutepuissance protectrice de l'Artémis d'Éphèse qui préside aux desseins des tyrans d'Éphèse, la proximité est grande. D'autre part, en 480, après la bataille de Salamine, c'est à Éphèse que Xerxès fait envoyer ses bâtards ${ }^{16}$, En 479 , a près la bataille de Platées, c'est un Éphésien, Dionysophanès, qui fait enterrer luimême le corps de Mardonios, le général Perse ${ }^{17}$.

Domination des despotes sur la Perse, domination des tyrans sur la cité d'Éphèse se ressemblent, comme se ressemblent la déesse dominatrice perse Ahura et la déesse dominatrice éphésienne qu'est Artémis.

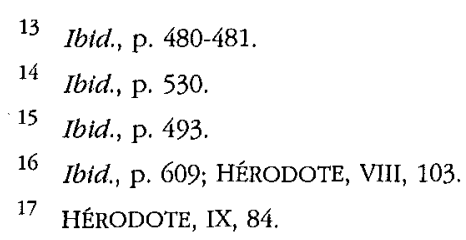




\section{L'époque de la démocratie à Éphèse}

À l'époque qui succède à la révolte manquée d'Ionie (494) et surtout au lendemain des défaites perses (Marathon 490, Salamine 480), les Éphésiens abandonnent le régime tyrannique (chute de Mélancomas) et se donnent une constitution démocratique plus ou moins inspirée des réformes athéniennes de Clisthène. L'on dépouille les nobles de la puissance qu'ils puisaient non dans les lois mais dans les coutumes et les mœurs, l'on prend des précautions d'une efficacité certaine contre toute tentative de pouvoir personnel, on s'inspire des doctrines mathématiques pythagoriciennes dans la distribution des pouvoirs, c'est désormais l'autorité souveraine de l'Ecclesia qui l'emporte, avec le tirage au sort des magistrats. Enfin l'on assiste à l'introduction de la loi clisthénienne sur l'ostracisme, qui permettait au peuple de prévenir tout attentat contre l'ordre établi en chassant quiconque serait soupçonné de nourrir pareil dessein. C'est d'ailleurs par cette loi que les Éphésiens, aux alentours de 475, allaient chasser l'homme politique qui présidait à leur destinée : le remarquable chef d'État démocrate qu'était Hermodore.

À cette conception politique nouvelle de la Justice s'associe parallèlement, comme à Athènes, une nouvelle représentation des dieux. Ces êtres de force, que l'lliade représentait comme des géants souvent querelleurs et méchants, égoïstes même quand ils rendent service, les voilà qui se métamorphosent. Ils gardent leurs traits physiques, cette apparence humaine que les artistes reproduisent sur les images destinées à orner leurs temples. Mais les dieux ont changé d'âme. Ils ont perdu leurs vices guerriers et sont devenus bons. Pour la première fois, leur puissance se justifie par leurs vertus, tout comme l'infortune des mortels s'explique par leurs erreurs et leurs fautes. Désormais les Olympiens se dressent en défenseurs des lois naturelles qui sont le fondement de l'harmonie universelle. Les dieux se sont moralisés. Les dieux vont jusqu'à faire régner la paix et l'équité dans le monde d'outre-tombe. Des dieux soumis à la Justice, à la Loi, à Dikè, à Nomos.

Tels sont les dieux qu'évoquent certains fragments d'Héraclite. Toutefois Héraclite réfute les insuffisantes conceptions religieuses qu'à ses yeux les dieux impliquent. Les dieux pacifiés et puissants que cite Héraclite, ce n'est point l'Artémis toute-puissante d'Éphèse jamais nommée. Mais ce sont Zeus, Dionysos, Hadès, Arès; derrière l'énoncé de certains fragments, l'on reconnaît encore Apollon et Déméter. Quant à Artémis, elle ne se trouve que très indirectement évoquée, en fonction de ses prêtres hubristiques, les Mages. Mais aucune de ces figures divines ne s'accorde avec la conception épurée du divin qu'envisage Héraclite. Quant aux lois démocratiques visant à égaliser les citoyens, elles ne trouvent point non plus l'assentiment d'Héraclite. Opposées qu'elles sont à un aristocratisme de la vraie valeur.

Ainsi à une constitution d'inspiration clisthénienne visant la justice se lie parallèlement une multiplicité de dieux plus ou moins soucieux de justice. 


\section{La critique politico-religieuse et la conception esquissée d'une royauté idéale selon Héraclite}

Les deux gouvernements historiques qu'Héraclite a successivement rencontrés, une tyrannie dominatrice et une démocratie égalitaire, ont un point commun : se préoccuper du bonheur matériel du peuple, de l'essor matériel de la cité entière.

Or Héraclite n'a que mépris pour le peuple et la populace et pour les joies matérielles. Les hommes du troupeau constituent, plus que d'autres, les " assoupis », les "endormis " ${ }^{18}$. Les hommes en masse se règlent démocratiquement sur ce qui se dit et s'entend généralement. «Qu'est leur intelligence, leur pensée viscérale? Ils se fient aux chanteurs populaires et prennent pour maitre la multitude, ne sachant pas que la plupart des hommes sont mauvais et que peu sont bons ${ }^{19}$. Les hommes du troupeau ne sont pas seulement des radicalement «endormis ». Leur désir de posséder reste mesquinement enfermé en une subjectivité oublieuse d'autrui. Les hommes mauvais du fragment 104 renvoient à toutes les formes de l'excès. La fin du fragment 29 est nette : «La plupart sont repus comme du bétail ». Deux métaphores, celle de l'âne et celle du porc en découlent. «Les ânes choisiraient la paille plutôt que l'or ». «Les porcs se complaisent dans la fange plutôt que dans l'eau pure ». À la recherche des plaisirs futiles se joint la course à l'argent : «Que la richesse à vous ne fasse pas défaut, Éphésiens, afin que vous soyez convaincus d'être des misérables ${ }^{20}$. Toutefois, ce n'est pas tant la richesse que ne pas savoir être au-dessus de leurs richesses, de ne pas savoir les mépriser qu'Héraclite reproche aux hommes de la foule.

Ce qu'Héraclite envisage comme étant le meilleur, c'est un pouvoir royal ferme. Le véritable homme politique, c'est celui qu'éclaire l'authentique philosophe. Le vrai philosophe, c'est le Gardien (pbulax) des secrets de l'univers. Le chef de la cité devra s'inspirer de lui. Comment dénommer le chef politique? Héraclite ne le dit pas explicitement, mais il convient de rapprocher la pensée et l'activité du Chef de celles du dieu régissant le monde, et dont il est une image sur terre. Or deux termes sont ici utilisés tour à tour par Héraclite : celui de «basileus » et celui d' «anax » pour désigner l'Organisateur de l'univers. Le fragment 53 s'exprime ainsi : «La guerre est le père de toutes choses, de toutes le Roi » et, retrouvant la terminologie homérique, le fragment 93, évoquant la figure du dieu Apollon, dit : «Le Maître (anax), dont l'oracle est celui de Delphes, ne dit ni ne cache, mais donne des signes ».

Ce pouvoir royal repose sur l'aristocratie du mérite, celle des guerriers et celle des authentiques penseurs. Dans la hiérarchie des habitants de la cité idéale d'Héraclite, après le philosophe sage et le politique juste, viennent se

\footnotetext{
18 HÉRACLITE, fr. 1 Diels-Kranz (Berlin, 195166).

19 HÉRACLITE, fr. 104 Diels-Kranz (trad. M. CONCHE, Paris, 1986).

20 Fr. 29, F. 9, F. 13 et fr. 125a Diels-Kranz.
} 
placer les «aristoi ». Que désigne ce terme des «meilleurs »? Ce sont d'abord et avant tout les guerriers protecteurs de la cité. Mais par aristoi, Héraclite n'envisage-t-il de désigner que les seuls guerriers? Ces meilleurs, ce sont les hommes de la gloire (kleos) ou, comme le dit encore le fragment 24 , les hommes de l'honneur (timê); ce sont les nobles de droit, qu'ils soient de haute naissance ou non. «En face de l'aristocratie de fait, et donc, d'une certaine façon, contre elle, Héraclite construit le type idéal d'une aristocratie de droit $»^{21}$.

La cité la meilleure, pour Héraclite, est une cité où l'aristocratie de l'intellect et l'aristocratie guerrière concourent à l'indépendance de la cité et à son essor intellectuel. Cité intellectualisée, moralisée, hiérarchisée : tel est l'idéal qu'Héraclite oppose et aux régimes tyranniques et aux régimes démocratiques. C'est en fonction d'un fondement théologique et métaphysique épuré ${ }^{22}$ que devrait se bâtir une politique rénovée, à la fois royale et aristocratique, de la cité idéale. Semblable doctrine, au demeurant, s'avère fort peu compréhensive à l'égard des besoins de la foule, celle des artisans et celle des commerçants.

Parallèlement, Héraclite s'oppose à toutes les variétés de religion que comporte aussi bien l'histoire d'Éphèse que l'histoire de la Perse ou l'histoire de la Grèce. Les prêtres certes s'élèvent au-dessus de la foule, mais leur pensée s'enferme dans l'illusoire. Il est toute une variété de doctrines religieuses qu'il convient de pourfendre. Tous ces multiples prêtres sont «errants dans la nuit ». On trouve d'abord à Éphèse des mages; ce sont des prêtres du mazdéîsme, c'est-à-dire de la religion dualiste de Zoroastre qui était devenue, à l'époque de Darius, la religion officielle de l'empire perse : un dieu suprême opposé à l'esprit destructeur lequel lui est subordonné. Autre culte : de façon non moins erronée, les Bacchants et les Bacchantes, adeptes de Dionysos, se livrent à l'orgie dionysiaque où la raison s'effondre: nuit encore de l'intelligence. Autres cultes : les initiés au culte de la Déméter éleusinienne ou les initiés à l'orphisme ${ }^{23}$.

Trois grands moments jalonnent les années 550-450 à Éphèse. On trouve d'abord la suprématie despotique de la déesse Artémis parallèlement au despotisme des tyrans. Plus tard, on rencontre une multiplicité de dieux au service de la justice, et parallèlement des magistrats démocrates épris de justice. Vient alors un troisième moment : celui de la critique philosophique héraclitéenne, aussi bien des idées religieuses et politiques de l'époque des tyrans, aussi bien des idées religieuses et politiques de l'époque de la démocratie. N'y aurait-il pas là comme une loi, qui se retouvera en bien des lieux ? L'on songe à l'Athènes où règnent les tyrans et le Zeus de l'Iliade, suivie de

21 CONCHE, op. cit. (n. 19), p. 121.

22 Fr. 32 Diels-Kranz : "L'Un, le Sage, ne veut pas et veut être appelé seulement du nom de Zeus "s.

23 F. $14 a$ Diels-Kranz. 
l'Athènes de Solon, de Pisistrate, de Clisthène, qui est aussi celle des dieux Olympiens, enfin, à l'époque de la cité idéale et à celle de la religion idéale : celles de la République et des Lois de Platon.

Université de Strasbourg II

Jean FRÈRE

14, rue Descartes

F - 67084 STRASBOURG Cedex 